\title{
HIV taken by STORM: Super-resolution fluorescence microscopy of a viral infection
}

\author{
Cândida F Pereira ${ }^{2,3,4 \dagger}$, Jérémie Rossy ${ }^{1 \dagger}$, Dylan M Owen ${ }^{1}$, Johnson Mak ${ }^{2,5,6^{*}}$ and Katharina Gaus ${ }^{1 *}$
}

\begin{abstract}
Background: The visualization of viral proteins has been hindered by the resolution limit of conventional fluorescent microscopes, as the dimension of any single fluorescent signal is often greater than most virion particles. Super-resolution microscopy has the potential to unveil the distribution of proteins at the resolution approaching electron microscopy without relying on morphological features of existing characteristics of the biological specimen that are needed in EM.

Results: Using direct stochastic optical reconstruction microscopy (dSTORM) to achieve a lateral resolution of 15$20 \mathrm{~nm}$, we quantified the 2-D molecular distribution of the major structural proteins of the infectious human immunodeficiency virus type 1 (HIV-1) before and after infection of lymphoid cells. We determined that the HIV-1 matrix and capsid proteins undergo restructuring soon after HIV-1 infection.
\end{abstract}

Conclusions: This study provides the proof-of-concept for the use of dSTORM to visualize the changes in the molecular distribution of viral proteins during an infection.

Keywords: HIV, Super-resolution microscopy, Electron microscopy, Viruses, Dynamic movement, Protein rearrangement

\section{Background}

The human immunodeficiency virus type 1 (HIV-1) is approximately spherical with a mean diameter of $125 \pm 14 \mathrm{~nm}$ [1-3]. Its main structural components are a lipid bilayer containing envelope glycoproteins, a matrix shell located beneath the viral lipid membrane and a capsid core with a cone shaped geometry [1-3]. After fusion of HIV-1 with the target cell, it is postulated that the matrix shell is retained at the plasma membrane while the capsid core undergoes a dramatic disassembly process that facilitates the reverse transcription of the viral genome [3-5]. Fluorescent labeling of HIV-1 proteins has provided valuable insights into their sub-cellular localization in infected cells [6,7]. However, the molecular mechanisms of cell entry and replication are difficult to detect since the viral particle is smaller than the resolution limit of conventional fluorescent microscopes. Electron microscopy techniques have provided structural

\footnotetext{
* Correspondence: johnson.mak@deakin.edu.au; k.gaus@unsw.edu.au ${ }^{\dagger}$ Equal contributors

${ }^{1}$ Centre for Vascular Research, University of New South Wales, Sydney, Australia

${ }^{6}$ Commonwealth Scientific and Industrial Research Organization, Australian Animal Health Laboratory, Geelong, Australia

Full list of author information is available at the end of the article
}

insights of HIV-1 $[1,2,7]$ but remain technically demanding and are prone to introduce artifacts.

The development of far-field super-resolution light microscopy methods such as stochastic optical reconstruction microscopy (STORM) [8] and photoactivatable localization microscopy (PALM) [9] enable the localization of individual photoswitchable protein-labeled molecules with a spatial resolution of tens of nanometers. The more recently developed direct STORM (dSTORM) combines standard immunocytochemistry, total internal reflection fluorescence (TIRF) microscopy and reversible photoswitching of conventional organic fluorochromes such as Cy5 and Alexa 647 to further improve the quality of signals [10]. This technique has a tremendous potential for the visualization of the molecular organization of viral proteins during an infection, particularly if tagging with fluorescent proteins compromises virus infectivity. Previously studies have used super-resolution techniques to visualize clusters of viral proteins artificially transfected into cell lines [11-13]. In this study we have used dSTORM to provide one of the first the molecular distribution of matrix and capsid proteins in infectious HIV-1 particles before and after a real infection of lymphoid cells.

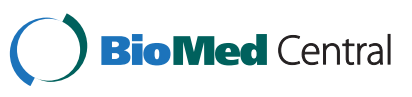




\section{Results and discussion}

We used ASTORM to visualize matrix and capsid proteins in cell-free HIV-1 virions and in infected lymphoid cells. Similar to previously describe $[7,14]$, we first generated HIV-1 particles that contained the green fluorescent protein-viral protein $\mathrm{R}$ fusion protein (HIVGFP-Vpr), which allows us to visualize particles with conventional fluorescence microscopy. The same virion preparation was then either evenly spread onto glass coverslips or used to infect lymphocytes. Importantly, non-internalized viral particles were removed from the surface of the target cells by pronase treatment twenty minutes after the virus was allowed to enter the target cells. This was confirmed by incubation of the target cells with an envelope-deficient HIV-1, which was unable to enter the target cells and therefore was cleaved by the pronase and consequently no viral protein could be detected in these samples (Figure 1). Therefore, all matrix and capsid protein clusters associated with the lymphocytes were internalized. Infected cells were fixed and plated onto glass coverslips by cytospin centrifugation. Both samples were immuno-stained with antibodies recognizing either the matrix or caspid protein.

We first compared conventional to super-resolution images. In TIRF images, HIV-1 proteins in infected Tlymphocytes appeared as bright punctuate structures (Figure 2a). In dSTORM, the stochastic activation of fluorophores allows the analysis of the point-spread function (PSF) of individual proteins. In addition to the $x-y$ localization, the fitting algorithms also return the localization precision, number of photons emitted per molecules, and background values associated with each molecule. These characteristics allow us to apply stringent conditions for single molecule detection [15] and standardize the image quality across the experimental conditions. When the molecular coordinates of the individual matrix proteins are plotted in an image, it becomes apparent that dSTORM revealed a greater heterogeneity in distribution of the same protein than in TIRF images (Figure 2b). The increase in resolution achieved with dSTORM is illustrated by the overlay of the two images with protein clusters in dSTORM
HIVGFP-Vpr
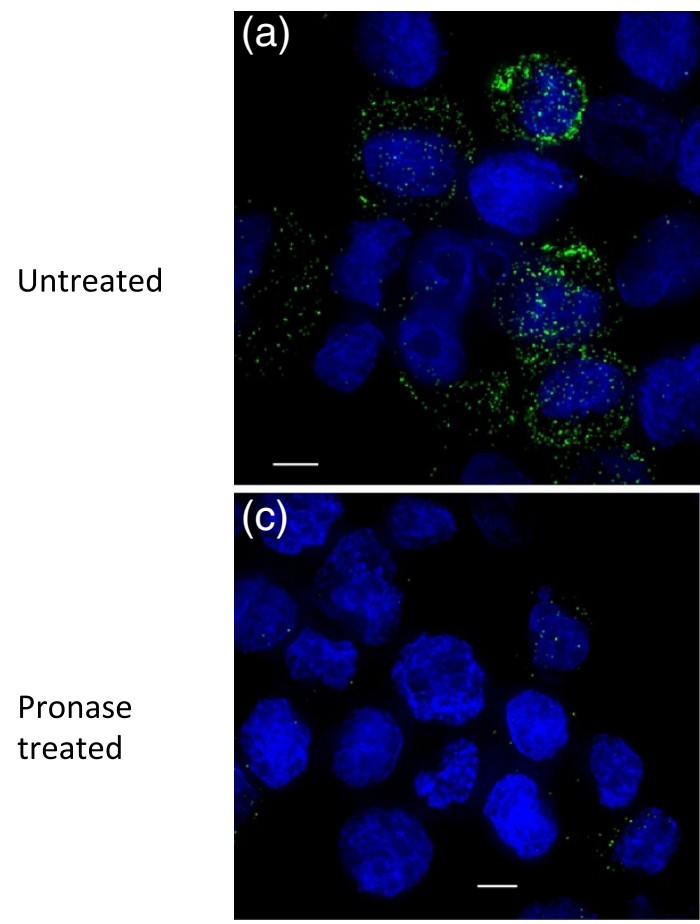

HIV $\triangle$ envGFP-Vpr
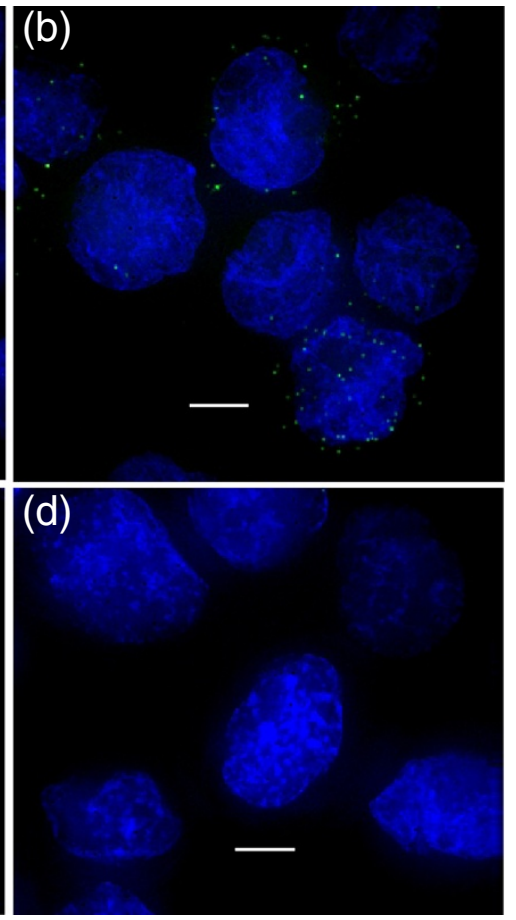

Figure 1 Pronase treatment removes non-internalized virus particles from the cell surface. MT-2 cells were infected with (a, c) HIVGFP-Vpr or

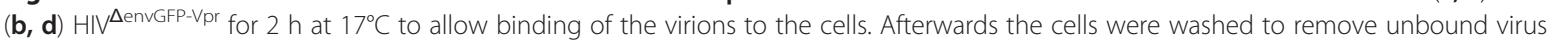
particles and incubated for $20 \mathrm{~min}$ at $37^{\circ} \mathrm{C}$ to allow virus entry into the cells. The samples were then split and half of the cells were incubated with PBS (a-b) while the other half was treated with pronase (c-d) to remove non-internalized virus particles. Subsequently, all samples were fixed, counterstained, mounted and visualized by widefield microscopy followed by deconvolution. GFP is shown in green and nuclei in blue. The provided images were derived from a volume compression of a z stack of 28 images taken at a 0.3- $\mu$ m step size. Scale bar, $5 \mu$ m. Images are representative of 3 independent experiments. 


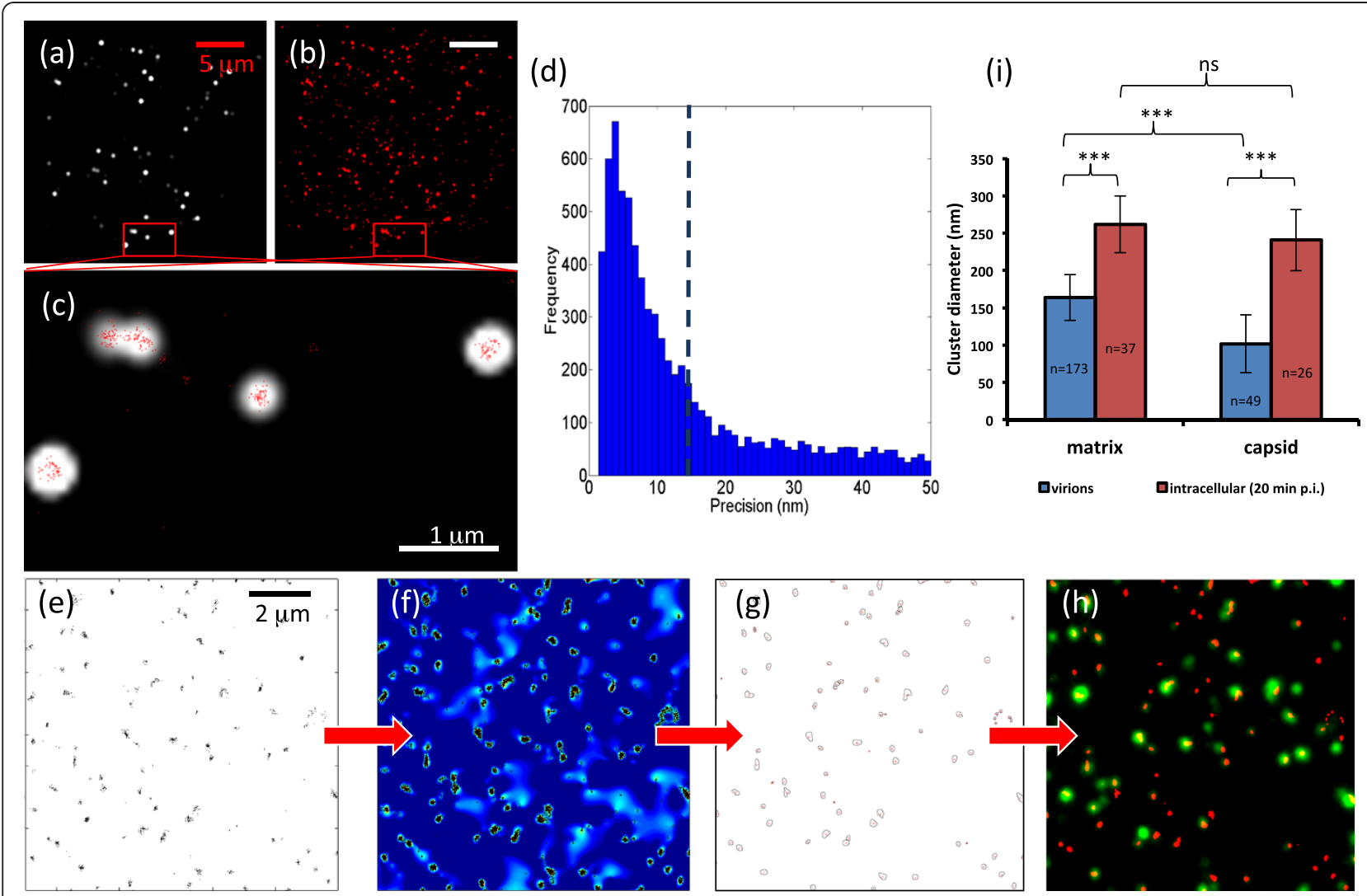

Figure 2 Super-resolution imaging of individual molecules of infectious HIV-1 before and after entry into lymphocytes. (a-c)

Conventional total internal reflection fluorescence (TIRF) image (a), corresponding dSTORM image (b) and overlay of the TIRF (white) and dSTORM (red) images (c) of the HIV-1 matrix protein 20 minutes after synchronized entry into the lymphoid cell line MT-2. (d) Histogram of the localization precision values of molecular coordinates localized by dSTORM corresponding to the data set shown in a-c. Localization precision corresponds to one sigma of the Gaussian distribution of the point spread function that is fitted to individual molecules and is also affected by photons and noise level. Dashed line indicates mean. (e-h) Cluster analysis of the matrix protein in cell-free virions based on Ripley's K-function converts the point distribution of molecular coordinates (e) into a cluster map with highly to less clustered regions colored red to blue (f). Cluster statistics such as number, size and associated molecules were extracted from thresholded images (g). By overlaying the TIRF image of GFP-Vpr (green) with the binary cluster map, the association of viral proteins with the reverse transcription complex after cell entry was quantified (h). Scale bars, $5 \mu m$ in panels in $\mathbf{a}-\mathbf{b}$; $1 \mu \mathrm{m}$ in panel $\mathbf{c}$ and $2 \mu \mathrm{m}$ in panels $\mathbf{e}-\mathbf{h}$. (i) Quantitative analysis of the diameter of the molecular clusters of capsid proteins in cell-free virions and 20 min post infection into MT-2 cells. Error bars represent the standard deviation of the mean from 26-173 clusters per sample from a representative from two experiments. ${ }^{* * *}=p<0.001$; ns $=$ non-significant.

appearing significantly smaller than the conventional image (Figure 2c). The molecular localization precision of the dSTORM approach was 15-20 nm (Figure 2d).

To quantify the distribution and heterogeneity of viral proteins, we used a variation on Ripley's K- function analysis [16]. As shown for the matrix protein in cell-free HIV-1 (Figure 2e-h), the single molecule dSTORM image was converted into a pseudo-colored cluster map that is based on the number of other molecules within a $50 \mathrm{~nm}$ radius, normalized to the overall particle density (Figure 2f). The contours describing each cluster were extracted and the number of clusters and cluster sizes determined (Figure 2g). To identify the replicating viruses that are travelling to the nucleus we used the GFP-Vpr fusion protein, which is incorporated into the virus particles and remains associated with the replicating viruses during intracellular trafficking [7]. GFP-Vpr was therefore used to identify 'double positive' viral protein clusters that were associated with the viral replication machinery and contained the matrix or capsid protein (Figure 2h).

This analysis allowed us to accurately measure the sizes of matrix and capsid protein clusters co-localized with GFP-Vpr in cell-free virons and in infected cells (Figure 2i). In cell-free virions, the size of the matrix protein clusters are within the expected range of 106$183 \mathrm{~nm}$ [1] and, as expected, the size of the capsid protein clusters are significantly smaller than the matrix protein clusters $(p<0.0001$, Figure $2 \mathrm{i})$. Twenty minutes post infection of $\mathrm{T}$ lymphocytes, the matrix protein 
clusters and the capsid protein clusters were similar in size (Figure 2i), which indicates that upon infection the capsid protein clusters showed a significantly large fractional increase in size $(236 \%)$ when compared with the matrix protein clusters. This single molecule imaging approach hence allowed us to follow the restructuring of the matrix shell and capsid core during infection, which may reflect the structural rearrangements that facilitate the HIV-1 reverse transcription process, such as virion uncoating.

\section{Conclusion}

We were able to quantify the size of the HIV-1 matrix shell and capsid core by dSTORM and these results were in agreement with the known HIV organization seen by EM $[1,2,17]$. Furthermore, this approach provided new information showing that upon cell entry, the size of the virion matrix shell and capsid core increased significantly when compared to cell-free HIV-1 virions, which indicates that the HIV particles underwent a dramatic rearrangement immediately after entry into the target cell. In summary, this study validates the use of dSTORM to assess the molecular distribution of viral proteins during the life-cycle of an infectious virus, and it opens up new possibilities to study the distribution and re-distribution of viral proteins at the early phase of viral infection.

\section{Methods}

\section{Cells and virus}

MT-2 cells (obtained through the AIDS Research and Reference Reagent Program, Division of AIDS, NIAID, NIH from D. Richman) $[18,19]$ were cultured in Rosewell Park Memorial Institute (RPMI) 1640 medium (Invitrogen) supplemented with $10 \% \mathrm{vol} / \mathrm{vol}$ heat-inactivated fetal calf serum (FCS; Invitrogen, Mount Waverley, Victoria, Australia) and penicillin/streptomycin. $293 \mathrm{~T}$ cells were maintained in Dulbecco's modified Eagle medium/ high modified (with $4500 \mathrm{mg} / \mathrm{l}$ dextrose and $4 \mathrm{mM} \mathrm{L}$ glutamine) medium (DMEM; Invitrogen), supplemented with $10 \%(\mathrm{vol} / \mathrm{vol})$ heat-inactivated cosmic calf serum (CCS; Hyclone, Tauranga, New Zealand), $100 \mathrm{U} / \mathrm{ml}$ of penicillin and $100 \mathrm{mg} / \mathrm{ml}$ of streptomycin (Invitrogen).

The pNL4-3 proviral DNA (obtained through the AIDS Research and Reference Reagent Program, Division of AIDS, NIAID, NIH from M. Martin [20]) contains the NL4-3 infectious molecular clone of HIV-1. The pNL4$3^{\Delta \text { env }}$ proviral DNA (obtained through the AIDS Research and Reference Reagent Program, Division of AIDS, NIAID, NIH from N. Landau [21,22]) contains an envelope defective-pNL4-3 molecular clone of HIV-1. HIV-1 particles were produced by poly(ethylenimine) (PEI; Polysciences Inc., Warrington, PA, USA) transfection of 293 T cells with pNL4-3 or pNL4-3 ${ }^{\Delta \text { env }}$ proviral DNA and GFP-Vpr plasmid (kindly provided by T. Hope,
Northwestern University) to generate GFP-Vpr-labeled HIV-1 (HIV ${ }^{\text {GFP-Vpr }}$ or HIV $\left.{ }^{\Delta \text { envGFP-Vpr }}\right)$. Forty hours posttransfection viral particles were purified, concentrated and quantified as previously described [14]. Briefly, supernatant from $293 \mathrm{~T}$ cells was filtered and viral particles were concentrated by ultracentrifugation through a $20 \%$ sucrose cushion at $100,000 \times \mathrm{g}$ for $1 \mathrm{~h}$ at $4^{\circ} \mathrm{C}$ using an L-90 ultracentrifuge (SW 41 rotor; Beckman, Fullerton, CA, USA) and virus pellets were resuspended in $1 \mathrm{x}$ phosphate buffered saline (PBS; Invitrogen) and quantified using a HIV-1 antigen (p24 CA) micro enzymelinked immunosorbent assay (ELISA) (Vironostika: Biomerieux, Boxtel, The Netherlands).

\section{Infection of lymphoid cells}

Synchronized infections were performed as described previously [23]. Briefly, MT-2 cells were infected with $\mathrm{HIV}^{\mathrm{GFP}-\mathrm{Vpr}}$ (normalized to $1000 \mathrm{ng}$ of p24 per million cells) by spinoculation at $17^{\circ} \mathrm{C}$ for $2 \mathrm{~h}$ at $1,200 \mathrm{x}$ g. Afterwards, the cells were washed twice with PBS to remove unbound virus and incubated with warm media at $37^{\circ} \mathrm{C}$, $5 \% \mathrm{CO} 2$ for $20 \mathrm{~min}$ to initiate infection. Afterwards, the cells were washed, treated with $2 \mathrm{mg} / \mathrm{ml}$ of protease from Streptomyces griseus (pronase E; Sigma-Aldrich, Castle Hill, NSW, Australia) for $10 \mathrm{~min}$ on ice and washed extensively with PBS containing 20\% FCS. The cells were then fixed with $4 \%$ formaldehyde (Polysciences) in $0.1 \mathrm{M}$ pipes buffer, $\mathrm{pH}$ 6.8, washed with PBS and cytospined onto glass coverslips. Cell-free viruses (same batch as used for the infection of lymphoid cells) were also fixed with formaldehyde in pipes buffer, evenly spread on glass slides to achieve optimal sample thickness, incubated at $4^{\circ} \mathrm{C}$ for $16 \mathrm{~h}$ and washed twice with PBS.

\section{Immunofluorescence staining}

Cells and virus were permeabilized and stained with mouse anti-matrix (SVM-33) antibody (MH-SVM33C9, ATCC, Manassas, VA (Akzo Nobel N.V.) or mouse anticapsid (AG3.0) antibody (obtained through the AIDS Research and Reference Reagent Program, Division of AIDS, NIAID, NIH from J. Allan) [24] and goat Cy5conjugated anti-mouse secondary antibody (Jackson ImmunoResearch, USA).

\section{Image acquisition and analysis}

Antibody stained cells were imaged in an oxygen scavenging buffer $(50 \mu \mathrm{g} / \mathrm{ml}$ glucose oxidase, $25 \mu \mathrm{g} / \mathrm{ml}$ horseradish peroxidase, $75 \mathrm{mM} \quad \beta$-mercaptoethylamine, $25 \mathrm{mM}$ Hepes, $25 \mathrm{mM}$ glucose, $5 \%$ glycerol in PBS, pH 8) in an open Chamlide chamber (Live Cell Instrument, Seoul, Korea). Cells were imaged with surface-immobilized $100 \mathrm{~nm}$ colloidal gold beads (BBInternational, 
Cardiff, UK) that allow correction for sample drift during the acquisition.

dSTORM images were acquired on a prototype PALM microscope (Carl Zeiss GmbH, Jena, Germany) with TIRF illumination. In dSTORM, the carbocyanine dye Cy5 is stochastically converted to a long-lived dark state ('off') when excited using $633 \mathrm{~nm}(15 \mathrm{~mW})$ laser radiation and switched back 'on' by exposure to low intensities of $488 \mathrm{~nm}(0.1-1 \mathrm{~mW})$ laser light when the sample is immersed in a oxygen depleted buffer containing a reducing agent [10]. By adjusting the intensity of the $488 \mathrm{~nm}$ laser, the density of fluoroescent molecules was approximately kept constant during acquisition and across samples. Images of 5-6 cells per sample from two different experiments were captured using an Andor iXon DU-897D EMCCD camera (Andor Technology Plc, Belfast, UK), giving a pixel size of $100 \mathrm{~nm}$ at the sample plane.

dSTORM images were reconstructed from a series of 20,000 TIRF images using Zeiss Zen software. Molecular clustering was analyzed using Getis and Franklins $2^{\text {nd }}$ order analysis as previously described [25]. Localization precision corresponds to one sigma of the Gaussian distribution of the point spread function that is fitted to individual molecules and is also affected by photons and noise level [16]. Data was cropped so as to exclude points with localization precision worse than $50 \mathrm{~nm}$. A $10 \times 10 \mu \mathrm{m}$ region is then selected for analysis and rendered into cluster maps with $7 \mathrm{~nm} /$ pixel resolution. Cluster maps were threshold to create a binary map from which only clusters that significantly overlapped with a TIRF image of GFP-Vpr were selected and analyzed using ImageJ [26].

\section{Pronase treatment efficiency}

MT-2 cells were infected with HIV ${ }^{\text {GFP-Vpr }}$ or $\mathrm{HIV}^{\Delta \text { envGFP- }}$ $\mathrm{Vpr}$ as described above and afterwards the samples were split. Half of the cells were treated with pronase to remove non-internalized virus as described above and half of the cells were incubated with PBS. The cells were then cytospined onto glass slides, counterstained with Hoechst 33258 (Invitrogen), mounted in Fluoromount-G (Electron Microscopy Sciences, Hatfield, PA) and images were captured in a $\mathrm{z}$ series on a charge-coupled device (CCD) camera (CoolSnap HQ; Photometrics, Tucson, AZ) through a $100 \times 1.4$ numerical aperture $(\mathrm{NA})$ oil immersion lens on a DeltaVision microscope (Applied Precision, Issaquah, WA) and deconvolved using softWoRx deconvolution software (Applied Precision).

\section{Statistical analysis}

Data derived from the diameter of 26-173 molecular clusters per sample was analyzed by paired two-tailed
Student's $t$ test. A $p$ value $<0.001$ was considered highly statistically significant for all tests.

\section{Competing interests}

The authors declare that they have no competing interests.

\section{Acknowledgments}

We acknowledge the financial support from the National Health and Medical Research Council of Australia (C.F.P., J.M., K.G.), the Australian Research Council (K.G., D.O., J.M.), Human Frontier Science Pro- gram (K.G.), the European Social Fund of the European Commission (C.F.P.) and the Portuguese Foundation for Science and Technology (C.F.P.). J.M. is a recipient of the Pfizer Foundation Fellowship and ARC Future Fellowship. We thank T. Hope for the GFP-Vpr plasmid and S. Turville for the MH-SVM33C9 purified hybridoma supernatant.

\section{Author details}

${ }^{1}$ Centre for Vascular Research, University of New South Wales, Sydney, Australia. ${ }^{2}$ Centre for Virology, Burnet Institute, Melbourne, Australia. ${ }^{3}$ Monash Micro Imaging, Clayton, Australia. ${ }^{4}$ Department of Medicine, Monash University, Clayton, Australia. ${ }^{5}$ School of Medicine, Deakin University, Geelong, Australia. ${ }^{6}$ Commonwealth Scientific and Industrial Research Organization, Australian Animal Health Laboratory, Geelong, Australia.

\section{Author's contributions}

CFP carried out the virus components of this study in the Mak lab (JM) at Burnet Institute; JR and DMO conducted the dSTORM imaging and analyses in the Gaus lab (KG) at UNSW. All authors contributed to the design, data interpretation and writing of the manuscript. All authors read and approved the final manuscript.

Received: 9 November 2011 Accepted: 2 May 2012

Published: 2 May 2012

\section{References}

1. Briggs JA, Grunewald K, Glass B, Forster F, Krausslich HG, Fuller SD: The mechanism of HIV-1 core assembly: insights from three-dimensional reconstructions of authentic virions. Structure 2006, 14:15-20.

2. Goto T, Nakai M, Ikuta K: The life-cycle of human immunodeficiency virus type 1. Micron 1998, 29:123-138.

3. Miller MD, Farnet CM, Bushman FD: Human immunodeficiency virus type 1 preintegration complexes: studies of organization and composition. J Virol 1997, 71:5382-5390.

4. Karageorgos L, Li P, Burrell C: Characterization of HIV replication complexes early after cell-to-cell infection. AIDS Res Hum Retroviruses 1993, 9:817-823.

5. Yamashita M, Perez O, Hope TJ, Emerman M: Evidence for direct involvement of the capsid protein in HIV infection of nondividing cells. PLOS Pathog 2007, 3:1502-1510.

6. Arhel N, Genovesio A, Kim KA, Miko S, Perret E, Olivo-Marin JC, Shorte S, Charneau P: Quantitative four-dimensional tracking of cytoplasmic and nuclear HIV-1 complexes. Nat Methods 2006, 3:817-824.

7. McDonald D, Vodicka MA, Lucero G, Svitkina TM, Borisy GG, Emerman M, Hope TJ: Visualization of the intracellular behavior of HIV in living cells. $J$ Cell Biol 2002, 159:441-452.

8. Rust MJ, Bates M, Zhuang X: Sub-diffraction-limit imaging by stochastic optical reconstruction microscopy (STORM). Nat Methods 2006, 3:793-795.

9. Betzig E, Patterson GH, Sougrat R, Lindwasser OW, Olenych S, Bonifacino JS, Davidson MW, Lippincott-Schwartz J, Hess HF: Imaging intracellular fluorescent proteins at nanometer resolution. Science 2006, 313:16421645.

10. Heilemann M, van de Linde S, Schuttpelz M, Kasper R, Seefeldt B, Mukherjee A, Tinnefeld $P$, Sauer M: Subdiffraction-resolution fluorescence imaging with conventional fluorescent probes. Angew Chem Int Ed Engl 2008, 47:6172-6176.

11. Hess ST, Gould TJ, Gudheti MV, Maas SA, Mills KD, Zimmerberg J: Dynamic clustered distribution of hemagglutinin resolved at $40 \mathrm{~nm}$ in living cell membranes discriminates between raft theories. Proc Natl Acad Sci U S A 2007, 104:17370-17375. 
12. Eckhardt M, Anders M, Muranyi W, Heilemann M, Krijnse-Locker J, Muller B: A SNAP-tagged derivative of HIV-1-a versatile tool to study virus-cell interactions. PLoS One 2011, 6:e22007.

13. Lehmann M, Rocha S, Mangeat B, Blanchet F, Uji IH, Hofkens J, Piguet V: Quantitative multicolor super-resolution microscopy reveals tetherin HIV1 interaction. PLoS Pathog 2011, 7:e1002456.

14. Pereira CF, Ellenberg PC, Jones KL, Fernandez TL, Smyth RP, Hawkes DJ, Hijnen M, Vivet-Boudou V, Marquet R, Johnson I, Mak J: Labeling of multiple HIV-1 proteins with the biarsenical-tetracysteine system. PLoS One 2011, 6:e17016.

15. Williamson DJ, Owen DM, Rossy J, Magenau A, Wehrmann M, Gooding JJ, Gaus K: Pre-existing clusters of the adaptor Lat do not participate in early T cell signaling events. Nat Immunol 2011, 12:655-662.

16. Owen DM, Rentero C, Rossy J, Magenau A, Williamson D, Rodriguez M, Gaus K: PALM imaging and cluster analysis of protein heterogeneity at the cell surface. J Biophotonics 2010, 3:446-454.

17. Welker R, Hohenberg H, Tessmer U, Huckhagel C, Krausslich HG: Biochemical and structural analysis of isolated mature cores of human immunodeficiency virus type 1. J Virol 2000, 74:1168-1177.

18. Harada S, Koyanagi Y, Yamamoto N: Infection of HTLV-III/LAV in HTLV-Icarrying cells MT-2 and MT-4 and application in a plaque assay. Science 1985, 229:563-566.

19. Haertle T, Carrera CJ, Wasson DB, Sowers LC, Richman DD, Carson DA: Metabolism and anti-human immunodeficiency virus-1 activity of 2-halo2',3'-dideoxyadenosine derivatives. J Biol Chem 1988, 263:5870-5875.

20. Adachi A, Gendelman HE, Koenig S, Folks T, Willey R, Rabson A, Martin MA: Production of acquired immunodeficiency syndrome-associated retrovirus in human and nonhuman cells transfected with an infectious molecular clone. J Virol 1986, 59:284-291.

21. He J, Choe S, Walker R, Di Marzio P, Morgan DO, Landau NR: Human immunodeficiency virus type 1 viral protein $R(V p r)$ arrests cells in the $G 2$ phase of the cell cycle by inhibiting p34cdc2 activity. J Virol 1995, 69:6705-6711.

22. Connor Rl, Chen BK, Choe S, Landau NR: Vpr is required for efficient replication of human immunodeficiency virus type-1 in mononuclear phagocytes. Virology 1995, 206:935-944.

23. O'Doherty U, Swiggard WJ, Malim MH: Human immunodeficiency virus type 1 spinoculation enhances infection through virus binding. J Virol 2000, 74:10074-10080

24. Simm M, Shahabuddin M, Chao W, Allan JS, Volsky DJ: Aberrant Gag protein composition of a human immunodeficiency virus type 1 vif mutant produced in primary lymphocytes. J Virol 1995, 69:4582-4586.

25. Perry GLW: SpPack:spatial point pattern analysis in Excel using Visual Basic for Applications (VBA). Environ Model Softw 2004, 19:559-569.

26. Abramoff MD, Magelhaes PJ, Ram SJ: Image Processing with ImageJ. Biophotonics Int 2004, 11:36-42

doi:10.1186/1743-422X-9-84

Cite this article as: Pereira et al:: HIV taken by STORM: Super-resolution fluorescence microscopy of a viral infection. Virology Journal 2012 9:84.

\section{Submit your next manuscript to BioMed Central and take full advantage of:}

- Convenient online submission

- Thorough peer review

- No space constraints or color figure charges

- Immediate publication on acceptance

- Inclusion in PubMed, CAS, Scopus and Google Scholar

- Research which is freely available for redistribution 